\title{
Adversity, cannabis use and psychotic experiences: evidence of cumulative and synergistic effects
}

Craig Morgan, Ulrich Reininghaus, Abraham Reichenberg, Souci Frissa, SELCOH study team, Matthew Hotopf and Stephani L. Hatch

\section{Background}

There is robust evidence that childhood adversity is associated with an increased risk of psychosis. There is, however, little research on intervening factors that might increase or decrease risk following childhood adversity.

\section{Aims}

To investigate main effects of, and synergy between, childhood abuse and life events and cannabis use on odds of psychotic experiences.

\section{Method}

Data on psychotic experiences and childhood abuse, life events and cannabis use were collected from 1680 individuals as part of the South East London Community Health Study (SELCOH), a population-based household survey.

\section{Results}

There was strong evidence that childhood abuse and number of life events combined synergistically to increase odds of psychotic experiences beyond the effects of each individually. There was similar, but weaker, evidence for cannabis use (past year).

\section{Conclusions}

Our findings are consistent with the hypothesis that childhood abuse creates an enduring vulnerability to psychosis that is realised in the event of exposure to further stressors and risk factors.

\section{Declaration of interest}

None.
Substantial evidence has accrued linking exposure to various forms of childhood adversity, particularly physical and sexual abuse, with both the occurrence of low-level psychotic experiences in adolescent and adult general population samples and (to a lesser degree) the onset of psychotic disorder. ${ }^{1-4}$ For example, the most comprehensive meta-analysis to date suggests that most of the forms of childhood adversity that have been studied (such as emotional, physical and sexual abuse; neglect; loss of a parent) are associated with around a two- to threefold increased risk of psychosis (broadly defined). These associations are evident irrespective of study design and there is some evidence of a dose-response effect, such that risk increases linearly with number of adversities.

Not all those exposed to adversity and abuse in childhood go on to develop psychotic experiences or psychotic disorder. Identifying the intervening factors that modify these relationships is an important next step in elucidating how and through which mechanisms negative experiences in childhood might contribute to risk. A number of possibilities have been proposed. For example, exposure to adversity and abuse in childhood may confer an enduring vulnerability to psychosis (and indeed other disorders) via deleterious effects on biological ${ }^{5}$ (such as hypothalamic-pituitaryadrenal axis function ${ }^{6}$ ) and psychological ${ }^{7}$ (such as affect and emotion $^{8}$ ) processes. This vulnerability may then become manifest as (initially) low-level psychotic experiences and, more rarely, psychotic disorder, in the event of exposure to further risk factors over time, such as, for example, subsequent life events and cannabis use. Both life events ${ }^{9}$ and cannabis use ${ }^{10}$ (to varying degrees) have been linked with psychosis and, as such, are strong candidate exposures that may have more pronounced effects in those with a pre-existing vulnerability. ${ }^{11,12}$ However, research on combined (or synergistic) effects of childhood adversity and other environmental exposures in psychosis remains underdeveloped. ${ }^{13}$

In light of this, using data from a large population-based household survey conducted in south-east London (UK), we sought to investigate, main and synergistic (combined) effects of childhood abuse, life events (ever and in the past year) and cannabis use (ever and in the past year) on low-level psychotic experiences. We hypothesised that: (a) childhood adversity (abuse), life events and cannabis use would be associated, singly and cumulatively, with psychotic experiences; and (b) childhood abuse would combine synergistically (on an additive scale) with (i) life events and (ii) cannabis use to increase odds of psychotic experiences. Our focus on low-level psychotic experiences follows much recent research and stems from the observation that such experiences are associated with an increased risk of both psychotic disorder and, to a lesser degree, other non-psychotic psychopathological outcomes, including suicidality. ${ }^{14-16}$ As such, studying risk factors for these experiences may tell us something about what exposures push some individuals along hypothesised continua of risk for psychosis and for mental disorder more broadly.

\section{Method}

The South East London Community Health Study (SELCoH) is a population-based household survey of physical and mental health conducted between 2008 and 2010 in the two London boroughs of Lambeth and Southwark. Overall, the two boroughs are areas with high levels of deprivation and ethnic diversity, with some pockets of relative wealth. Full details of the methods and primary descriptive outcomes are reported in Hatch et al. ${ }^{17,18}$

\section{Participants}

In brief, a random sample of households within the catchment area was identified using the Small User Postcode Address File (PAF; Royal Mail Address Management Unit, Sunderland, www. poweredbypaf.com/end-user/products/data-products/paf-raw-data/), which has near complete coverage of private households in the UK. 
Introductory letters were sent to all selected households, which were then visited up to four times at different times of the day and week, and when contact was made informed consent was sought from as many eligible members of the household as possible. All members of the household aged 16 years or over were eligible to participate. Ethical approval was received prior to the start of the study from King's College London Research Ethics Committee (CREC/07/08-152) and all participants provided informed consent.

\section{Data collection}

Data across a range of domains (including sociodemographic characteristics, socioeconomic status, physical and mental health, adversity and life events in childhood and adulthood and substance use) were collected using a computer-assisted interview schedule, which was piloted to establish reliability, validity and feasibility prior to the start of the main survey.

\section{Psychotic experiences}

The Psychosis Screening Questionnaire (PSQ) ${ }^{19}$ was used to elicit information on psychotic experiences. The PSQ is an intervieweradministered questionnaire that assesses psychotic experiences in the preceding year and comprises five sections covering hypomania, thought disorder, paranoia, strange experiences and hallucinations. Each section has an initial probe, followed by secondary questions that are designed to establish the psychotic quality of experiences. The PSQ has been validated in two national surveys in the UK. ${ }^{20,21}$ As we were specifically interested in psychotic experiences, items on hypomania were discarded. Following previous studies, ${ }^{22}$ in the analyses presented here, we primarily compared those who endorsed one or more secondary question in the remaining four domains on the PSQ with those who did not. Analyses were repeated for paranoia and hallucinations separately.

\section{Childhood exposures and life events}

Information on exposure to physical and sexual abuse and nine life events (see Table 3) ever and in the past year was elicited using single-item questions (for example for physical abuse: 'When growing up, did anyone hit you so hard that it left bruises or marks?' For sexual abuse: 'When growing up, did anyone who was responsible for your care - or someone else who was at least 5 years older than you - ever sexually abuse you?). ${ }^{23}$

\section{Socioeconomic indicators, substance use and common mental disorders}

The interview schedule included items on sociodemographic and economic indicators and substance use ever and in the past year (including cannabis). Ethnicity was self-ascribed. Social class was defined according to the Registrar General's Classification. ${ }^{24}$ Symptoms of common mental disorder were assessed with the Clinical Interview Schedule - Revised (CIS-R). ${ }^{25}$

\section{Data analysis}

To assess main effects, we used logistic regression to quantify associations between childhood exposures, life events, cannabis use and psychotic experiences, while adjusting for age, gender, ethnicity, education, social class (a summary indicator of socioeconomic position) and, as appropriate, cannabis use. On the assumption that risk factors add in their effects, presence of synergy between two (or more) factors is indicated by positive deviations from additivity. ${ }^{26}$ To examine whether there was evidence that childhood abuse and (a) life events and (b) cannabis use combined synergistically to increase odds of psychotic experiences, we tested for departure from additivity using interaction contrast ratios (ICRs), as described by Schwartz et $a l^{27}$ and $\mathrm{Knol}$ et $a .^{28}$ This approach allows use of odds ratios (ORs) derived from logistic models to estimate the relative excess risk as a result of synergy for combinations of dichotomous, ordinal and continuous exposures (i.e. ICR $=\mathrm{OR}_{\text {exposure } \mathrm{A} \& \text { exposure } \mathrm{B}-}$ $\left.\mathrm{OR}_{\text {exposure A only }}-\mathrm{OR}_{\text {exposure B only }}+1\right)$. In this model, positive deviation from additivity is indexed by an ICR greater than zero. Therefore, following Knol $\mathrm{et} \mathrm{al}^{28}$ to test our hypotheses on synergistic effects we first entered abuse, life events (or cannabis use) and the product of abuse and life events (or cannabis use) as independent variables in logistic models, with psychotic experiences as the dependent variable and age, gender, ethnicity, education, social class and, as appropriate, cannabis use as potential confounders. Using the odds ratios derived from these models for abuse, life events (or cannabis use), and abuse and life events (or cannabis use) combined, we calculated ICRs (i.e. ICR $=\mathrm{OR}_{\text {abuse \& life events (or }}$ cannabis use) $-\mathrm{OR}_{\text {abuse }}-\mathrm{OR}_{\text {life events (or cannabis use) }}+1$ ). Confidence intervals and $P$-values for ICRs were generated using the nlcom procedure in Stata version 11 (Windows). Within epidemiology, a strong case has been made that additive models provide the best representation of synergy, ${ }^{27,29}$ and that they are the most useful from a public health perspective. ${ }^{30}$ Analyses of main and synergistic effects, completed using Stata Version 11, accounted for clustering by household and were weighted for non-response within households using the relevant survey commands in Stata (see Hatch $e t a l^{17}$ for further details on weights).

\section{Results}

\section{Psychotic experiences}

In total, 2070 private households were contacted, of which 1075 (51.9\%) had at least one member who agreed to participate. Of the 2359 eligible individuals within these households, 1698 (71.9\%) agreed to be interviewed (mean number of participants per household 2.7 , s.d. $=1.2$ ). The sample was broadly representative of the catchment area population on basic demographic and socioeconomic characteristics (online Table DS1). Data from the PSQ was missing for 10 participants and 8 who reported having a current or past diagnosis of psychosis were excluded from the analyses presented here, giving a sample of 1680 (mean age 39, s.d. $=16.9$, men $n=734,44 \%)$. The 1 -year weighted prevalence of psychotic experiences, defined as a positive response to a secondary probe on the PSQ, was $17.9 \%$ (95\% CI 16.0-19.9, $n=315$ ), which was similar to what we found previously in a smaller study ${ }^{22}$ within the same catchment area $(18.8 \%$, online Table DS2).

The most commonly reported experience was paranoia $(n=237,13.2 \%)$.

\section{Markers of social disadvantage and isolation}

In line with previous studies, reporting a psychotic experience was associated with a range of demographic and socioeconomic variables, including younger age, ethnicity other than White British and low educational achievement (Table 1, see online Table DS3 for a more detailed version). Across a number of markers, those who reported a psychotic experience were more disadvantaged and isolated (i.e. more likely to be: unemployed, in rented housing, in receipt of benefits, in debt, in lower social class groups and single; Table 1 and online Table DS3). Further, those who endorsed a psychotic experience ( $v$. those who did not) were almost five times more likely to meet CIS-R threshold criteria for a common mental disorder (adjusted $\mathrm{OR}=4.99$, 95\% CI 3.75-6.62) (Table 1). In light of increasing evidence that symptoms of common mental disorder mediate or lie on a path 


\begin{tabular}{|c|c|c|c|c|}
\hline & \multirow[b]{2}{*}{ Total $n(\%)$} & \multicolumn{3}{|c|}{ Any psychotic experience(s) $(n=315)$} \\
\hline & & Yes, $n(\%)$ & Unadjusted OR & Adjusted OR $(95 \% \mathrm{Cl})^{\mathrm{b}}$ \\
\hline \multicolumn{5}{|l|}{ Gender } \\
\hline Male & 734 (33.4) & $147(19.1)$ & 1.00 & 1.00 \\
\hline Female & $946(66.6)$ & $168(17.2)$ & 0.88 & $0.88(0.69-1.13)$ \\
\hline \multicolumn{5}{|l|}{ Age, years } \\
\hline $16-29$ & $572(28.7)$ & $126(21.9)$ & 1.45 & $1.39(1.06-1.81)$ \\
\hline 30 and above & $1108(71.2)$ & $189(16.2)$ & 1.00 & 1.00 \\
\hline \multicolumn{5}{|l|}{ Ethnicity } \\
\hline White British & $1043(63.7)$ & $169(14.8)$ & 1.00 & 1.00 \\
\hline Other & $636(36.3)$ & $145(23.1)$ & 1.72 & $1.67(1.27-2.19)$ \\
\hline \multicolumn{5}{|l|}{ Education } \\
\hline No qualifications & $223(16.7)$ & $51(21.1)$ & 1.00 & 1.00 \\
\hline GCSES & $327(20.1)$ & $66(18.3)$ & 0.84 & $0.70(0.45-1.09)$ \\
\hline A-levels & $420(23.6)$ & $98(23.3)$ & 1.12 & $0.91(0.59-1.40)$ \\
\hline University & $691(39.7)$ & $94(12.8)$ & 0.55 & $0.52(0.34-0.78)$ \\
\hline \multicolumn{5}{|l|}{ Social class ${ }^{c}$} \\
\hline I and || & $505(28.4)$ & $66(12.6)$ & 1.00 & 1.00 \\
\hline$\| I N M$ and IIIM & 275 (15.3) & $50(17.1)$ & 1.43 & $1.22(0.79-1.89)$ \\
\hline IV and V & $164(10.0)$ & $28(16.8)$ & 1.39 & $1.23(0.74-2.05)$ \\
\hline Unclassified: unemployed & $162(9.1)$ & $53(32.1)$ & 3.28 & $2.93(1.87-4.58)$ \\
\hline Unclassified: other & 538 (37.2) & $110(20.5)$ & 1.60 & $1.44(1.00-4.58)$ \\
\hline \multicolumn{5}{|l|}{ Cannabis use } \\
\hline Never used & $855(55.2)$ & $141(15.6)$ & 1.00 & 1.00 \\
\hline Used, not in past year & $525(29.6)$ & $87(16.2)$ & 1.05 & $1.26(0.92-1.75)$ \\
\hline Used in past year & $300(15.1)$ & $87(29.6)$ & 2.28 & $2.47(1.73-3.53)$ \\
\hline \multicolumn{5}{|l|}{ Any common mental disorder } \\
\hline No & $1231(72.9)$ & $145(10.9)$ & 1.00 & 1.00 \\
\hline Yes & $444(27.2)$ & $168(36.5)$ & 4.70 & $4.99(3.75-6.62)$ \\
\hline \multicolumn{5}{|l|}{ Common mental disorder } \\
\hline No & $1233(73.0)^{\mathrm{d}}$ & $146(11.0)$ & 1.00 & 1.00 \\
\hline Mixed anxiety and depression & $127(7.7)$ & $45(33.6)$ & 4.11 & $4.43(2.89-6.81)$ \\
\hline Depression & $188(11.8)$ & $76(40.3)$ & 5.49 & $5.84(4.04-8.46)$ \\
\hline Anxiety related & $127(7.5)$ & $46(36.2)$ & 4.00 & $4.23(2.80-6.41)$ \\
\hline
\end{tabular}

from stress to psychosis, we did not treat common mental disorders as potential confounders in our analyses. In subsequent planned analyses, we will test mediation via common mental disorder in this sample.

\section{Main effects}

\section{Childhood}

We found strong evidence that the odds of reporting a psychotic experience were higher in those who, during childhood, had: (a) been physically abused (adjusted OR $=2.17$ ); and (b) been sexually abused (adjusted $\mathrm{OR}=2.13$; Table 2). The majority of those who reported sexual abuse also reported physical abuse $(n=46$, $58 \%$ ). When both physical and sexual abuse were considered together, there was evidence that the association with psychotic experiences was highest for those exposed to both (adjusted $\mathrm{OR}=3.24$; Table 2).

\section{Lifetime and past year exposure to life events}

In our sample, lifetime exposure to at least one of the nine life events enquired about was almost ubiquitous $(89 \%, n=1478)$ and $25 \%(n=422)$ reported exposure to at least one event in the preceding year. We found strong evidence that almost all the included life events, both lifetime and in the past year, were associated with between around a two- and fourfold increased likelihood of endorsing psychotic experiences (Table 3). Notably, for most events unadjusted and adjusted odds ratios were higher for reported exposure in the past year compared with ever, suggesting in general a greater impact the more proximal the life events. Further, the strongest effects in the past year were for 'been in a serious accident', 'witnessed violence', 'been attacked' and 'injured with a weapon'. What is notable is that each of these events has a quality of threat or intrusiveness (i.e. intrusion into the personal space and integrity of the individual).

Further, to examine the potential cumulative impact of lifetime exposure to life events, we created a simple index by summing the number of events participants reported (range 0-9; for the analyses, we combined those with five or more events ( $n=236,14 \%)$ into a single category). In doing this, we found very strong evidence of a linear relationship between number of events and odds of psychotic experiences, a pattern suggestive of a cumulative impact (Fig. 1(a)). When we narrowed the focus to events that had occurred in the preceding year and created a similar index (range $0-5$; for this analyses we combined those with three or more events $(n=32,1.9 \%)$ into a single category), this pattern was stronger still (Fig. 1(b)).

\section{Cannabis use}

Those who reported a psychotic experience were more likely to have used cannabis in the preceding year (adjusted $\mathrm{OR}=2.47$ ). There was, however, no evidence of an association between cannabis use prior to the preceding year and psychotic experiences (adjusted $\mathrm{OR}=1.26)($ Table 1$)$. 


\begin{tabular}{|c|c|c|c|c|c|}
\hline \multirow[b]{2}{*}{ When growing up . } & \multirow[b]{2}{*}{ Total $n(\%)$} & \multicolumn{4}{|c|}{ Any psychotic experience(s) } \\
\hline & & Yes, $n(\%)$ & Unadjusted OR & Adjusted OR $1^{\mathrm{b}}(95 \% \mathrm{Cl})$ & Adjusted OR $2^{\mathrm{C}}(95 \% \mathrm{Cl})$ \\
\hline \multicolumn{6}{|c|}{ Hit hard (physical abuse) } \\
\hline No & $1268(77.3)$ & $187(14.2)$ & 1.00 & 1.00 & 1.00 \\
\hline Yes & $402(22.7)$ & $127(30.6)$ & 2.66 & $2.50(1.89-3.29)$ & $2.17(1.62-2.89)$ \\
\hline \multicolumn{6}{|l|}{ Sexual abuse } \\
\hline No & $1584(94.8)$ & $283(17.0)$ & 1.00 & 1.00 & 1.00 \\
\hline Yes & $79(5.2)$ & $26(31.2)$ & 2.22 & $2.47(1.47-4.16)$ & $2.13(1.28-3.56)$ \\
\hline \multicolumn{6}{|l|}{ Hit hard or sexual abuse } \\
\hline No & $1229(75.1)$ & $176(13.8)$ & 1.00 & 1.00 & 1.00 \\
\hline Sexual abuse only & $33(2.2)$ & $7(20.6)$ & 1.62 & $1.84(0.75-4.53)$ & $1.79(0.78-4.11)$ \\
\hline Hit hard only & $354(19.7)$ & 107 (29.3) & 2.59 & $2.40(1.79-3.20)$ & $2.13(1.57-2.89)$ \\
\hline Both & $46(3.0)$ & 19 (39.1) & 4.01 & $4.12(2.15-7.89)$ & $3.24(1.68-6.25)$ \\
\hline $\begin{array}{l}\text { a. All percentages are wei } \\
\text { b. Adjusted for age, gende } \\
\text { c. Adjusted for age, gende }\end{array}$ & $\begin{array}{l}\text { bold signify } P \text { - } \\
\text { ation, social } \mathrm{Cl}\end{array}$ & nnabis & & & \\
\hline
\end{tabular}

\section{Synergistic effects of childhood abuse} and (a) life events and (b) cannabis use

We next examined whether there was evidence for synergistic effects of abuse and (a) life events and (b) cannabis use on odds of psychotic experiences. For these analyses, we combined exposure to either physical or sexual abuse into a dichotomous variable of exposed to either form of abuse or not and modelled life events as ordinal variables (ever and past year) using our indices described earlier.

\section{Table 3 Any psychotic experience(s) by lifetime and past year exposure to life events ${ }^{a}$}

\begin{tabular}{|c|c|c|c|c|c|}
\hline & \multirow[b]{2}{*}{ Total $n(\%)$} & \multicolumn{4}{|c|}{ Any psychotic experience(s) } \\
\hline & & Yes, $n(\%)$ & Unadjusted OR & Adjusted OR $1^{\mathrm{b}}(95 \% \mathrm{Cl})$ & Adjusted OR $2^{\mathrm{C}}(95 \% \mathrm{Cl})$ \\
\hline \multicolumn{6}{|c|}{ Separated from partner } \\
\hline No & $1061(62.7)$ & $168(14.8)$ & 1.00 & 1.00 & 1.00 \\
\hline Ever & $537(33.3)$ & $121(21.4)$ & 1.56 & $1.74(1.29-2.34)$ & $1.57(1.14-2.15)$ \\
\hline Past year & $73(4.0)$ & $25(37.1)$ & 3.38 & $3.06(1.85-5.04)$ & $2.36(1.36-3.99)$ \\
\hline \multicolumn{6}{|c|}{ Loved one died } \\
\hline No & $814(45.0)$ & $127(15.6)$ & 1.00 & 1.00 & 1.00 \\
\hline Ever & 700 (45.4) & $144(19.0)$ & 1.33 & $1.46(1.10-1.95)$ & $1.46(1.08-1.97)$ \\
\hline Past year & $156(9.7)$ & $43(26.8)$ & 2.08 & $2.07(1.34-3.20)$ & $2.02(1.26-3.24)$ \\
\hline \multicolumn{6}{|c|}{ No money for rent } \\
\hline No & $1561(93.6)$ & $274(16.9)$ & 1.00 & 1.00 & 1.00 \\
\hline Ever & $97(5.7)$ & $36(33.7)$ & 2.50 & $2.64(1.64-4.26)$ & $1.92(1.16-3.16)$ \\
\hline Past year & $13(0.6)$ & $4(30.0)$ & 2.11 & $1.83(0.52-6.46)$ & $0.95(0.23-3.97)$ \\
\hline \multicolumn{6}{|c|}{ Been in serious accident } \\
\hline No & $1290(78.2)$ & $213(15.8)$ & 1.00 & 1.00 & 1.00 \\
\hline Ever & $353(20.2)$ & $90(23.9)$ & 1.67 & $1.68(1.25-2.27)$ & $1.61(1.18-2.19)$ \\
\hline Past year & $28(1.6)$ & $11(46.5)$ & 4.64 & $4.75(1.98-11.35)$ & $4.07(1.72-9.64)$ \\
\hline \multicolumn{6}{|c|}{ Witnessed violence } \\
\hline No & $921(58.4)$ & $110(11.6)$ & 1.00 & 1.00 & 1.00 \\
\hline Ever & 603 (34.2) & $149(25.0)$ & 2.55 & $2.52(1.88-3.38)$ & $2.20(1.62-2.99)$ \\
\hline Past year & $146(7.4)$ & $55(35.7)$ & 4.24 & $3.92(2.61-5.89)$ & $3.31(2.18-5.06)$ \\
\hline \multicolumn{6}{|c|}{ Victim of crime } \\
\hline No & $1033(62.5)$ & $160(15.5)$ & 1.00 & 1.00 & 1.00 \\
\hline Ever & $563(33.6)$ & $132(23.5)$ & 1.59 & $1.76(1.34-2.33)$ & $1.59(1.19-2.13)$ \\
\hline Past year & $75(3.9)$ & $22(29.3)$ & 2.04 & $1.94(1.08-3.48)$ & $1.55(0.87-2.76)$ \\
\hline \multicolumn{6}{|l|}{ Attacked } \\
\hline No & $1172(71.6)$ & $164(13.3)$ & 1.00 & 1.00 & 1.00 \\
\hline Ever & $436(25.3)$ & $123(27.8)$ & 2.51 & $2.59(1.94-3.4)$ & $2.32(1.72-3.11)$ \\
\hline Past year & $61(3.1)$ & $26(41.8)$ & 4.68 & $4.52(2.63-7.77)$ & $3.53(2.05-6.09)$ \\
\hline \multicolumn{6}{|c|}{ Injured with weapon } \\
\hline No & 1475 (89.2) & $237(15.6)$ & 1.00 & 1.00 & 1.00 \\
\hline Ever & $179(10.0)$ & $70(36.8)$ & 3.15 & $3.28(2.28-4.71)$ & $2.59(1.77-3.78)$ \\
\hline Past year & $17(0.8)$ & $7(40.9)$ & 3.74 & $3.21(1.13-9.10)$ & $2.46(0.86-6.98)$ \\
\hline \multicolumn{6}{|c|}{ Been in war zone } \\
\hline No & $1460(86.2)$ & $275(18.2)$ & 1.00 & 1.00 & 1.00 \\
\hline Ever & $204(13.5)$ & 35 (17.2) & 0.82 & $0.85(0.56-1.30)$ & $0.69(0.43-1.08)$ \\
\hline Past year & $6(0.3)$ & $3(47.6)$ & 4.10 & $2.72(0.61-12.16)$ & $1.88(0.38-9.31)$ \\
\hline $\begin{array}{l}\text { a. All percentaz } \\
\text { b. Adjusted for } \\
\text { c. Adjusted for }\end{array}$ & $\begin{array}{l}\text { oold signify P } \\
\text { tion, social }\end{array}$ & Innabis use & & & \\
\hline
\end{tabular}


(a)

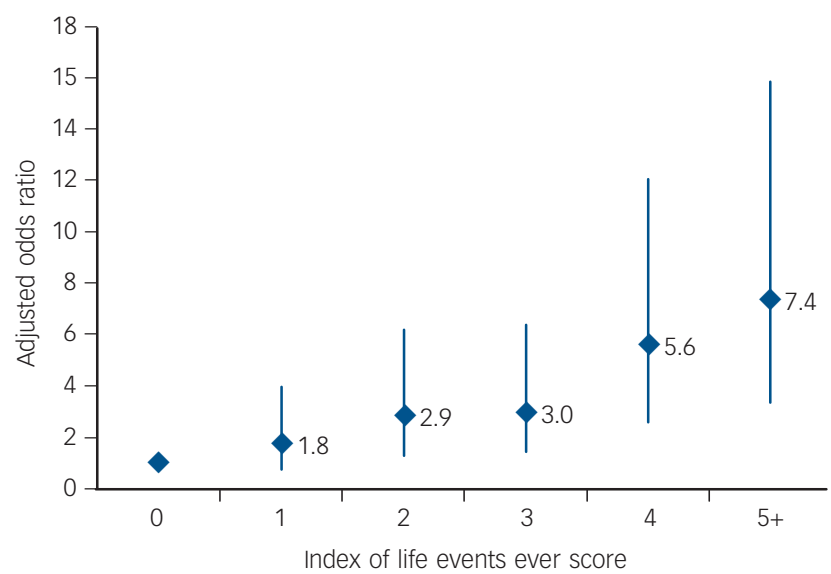

(b)

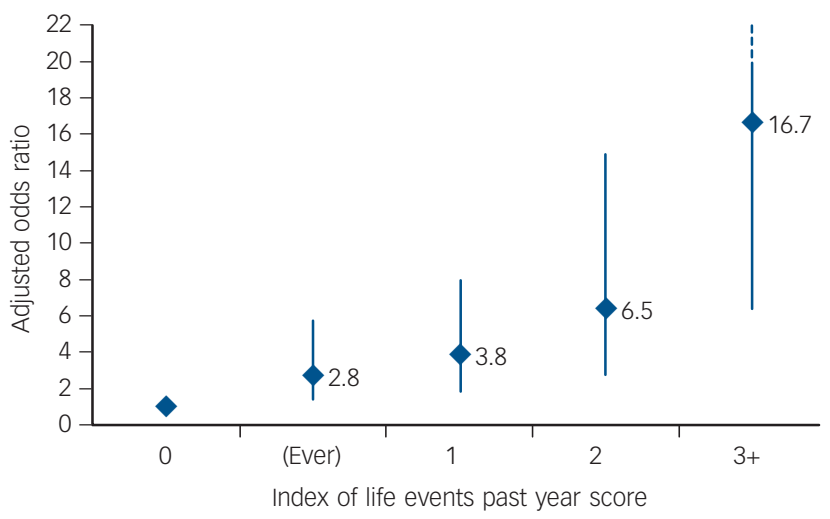

Fig. 1 Adjusted odds ratios (ORS) with 95\% confidence intervals for any psychotic experience(s) by indices of life events: (a) ever, (b) in past year.

Odds ratio adjusted for age, gender, ethnicity, education, social class and cannabis use. Presence of each event scores 1 and these are summed to create a simple index of exposure to the listed life events: in (a) for 'ever', with a range from 0 to 9 , the numbers scoring 5 and above were relatively small and so were combined in the final category of $5+$; in (b) for 'past year', with a range from 0 to 5 , the numbers scoring 3 and above were relatively small and so were combined in the final category of $3+$. When the linear effect of the index was modelled, there was strong evidence of linear effect: (a) adjusted $\mathrm{OR}=1.45,95 \% \mathrm{Cl} 1.32-1.59$, indicating an average increase in odds of psychotic experiences of 1.45 for every unit increase in number of life events; (b) adjusted $\mathrm{OR}=1.70$ ( $95 \% \mathrm{Cl} 1.44-2.00)$, indicating an average increase in odds of psychotic experiences of 1.70 for every unit increase in number of life events.
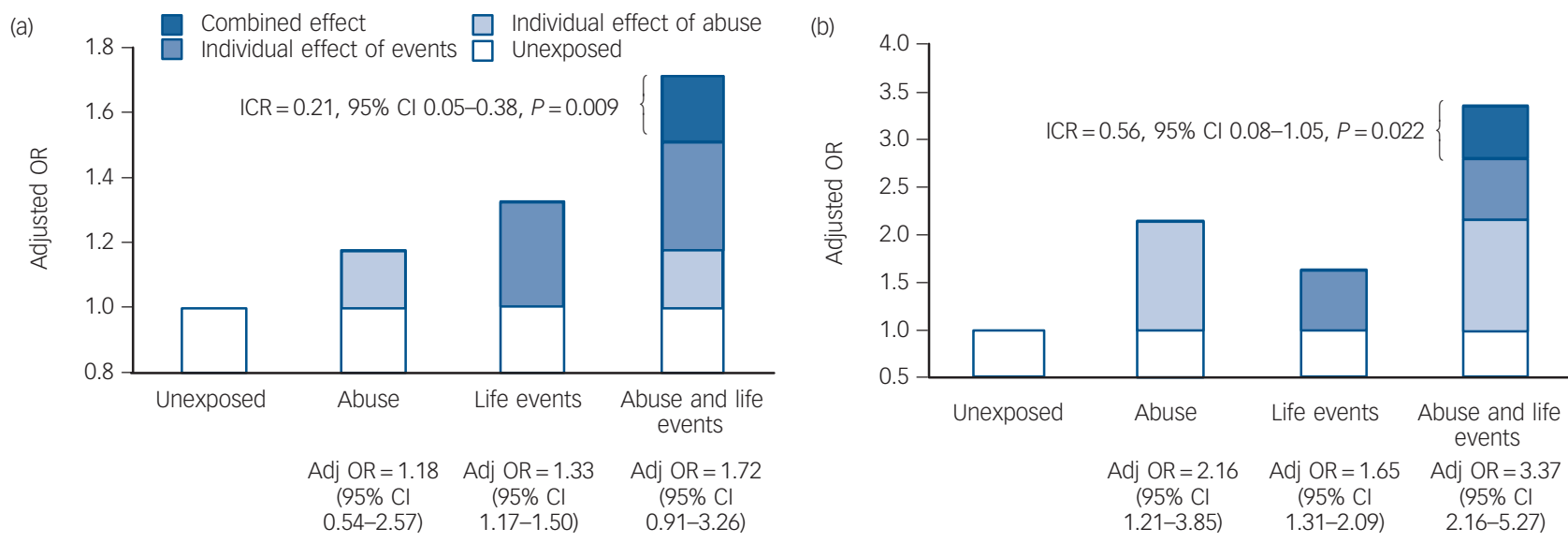

Fig. 2 Synergistic effects of abuse and life events: (a) ever, (b) in past year

Odds ratio (OR) adjusted for age, gender, ethnicity, education, social class and cannabis use. Adj, adjusted; IRC, interaction contrast ratio.

To begin with, abuse was strongly associated, in a linear regression, with an increased number of life events (number of events, ever: unadjusted $\beta=1.24,95 \%$ CI $1.07-1.42$; number of events, past year: unadjusted $\beta=0.33,95 \%$ CI $0.23-0.42$ ) and, in a logistic regression, with cannabis use ever (unadjusted $\mathrm{OR}=1.35,95 \%$ CI $1.04-1.75)$ and in the year prior to interview (unadjusted OR $=2.10,95 \%$ CI $1.55-2.85$ ).

When we considered abuse and life events, first using our index of life events (ever), there was strong evidence of synergy. The effect for abuse alone was 1.18 , a statistically non-significant effect at $P=0.05$, and for life events ever (modelled as an ordinal exposure) it was 1.33 , indicating an increase in odds of psychotic experiences of around $30 \%$ for each additional life event. The combined effect (adjusted OR $=1.72$ ), however, was greater than the sum of these individual effects: $\mathrm{ICR}=0.21$, 95\% CI $0.05-0.38, P=0.01$, (Fig. 2(a)). An ICR of 0.21 indicates that the odds ratio for psychotic experiences in those exposed to abuse is 0.21 higher with each additional life event than if there was no synergy between abuse and life events.
When we repeated these analyses using our index of life events (past year), evidence of synergy was stronger still, with effects of 2.16 for abuse and 1.65 for life events (past year), and a combined effect of 3.37 and an ICR of 0.56 (95\% CI $0.08-1.05, P=0.02)$ (Fig. 2(b))

For abuse and cannabis use in the past year, there was suggestive evidence that both combined synergistically to increase odds of psychotic experiences (Fig. 3). The adjusted odds ratio for those exposed to abuse and who used cannabis in the year before assessment was 5.54, compared with odds ratios of 2.04 for those exposed to abuse only and 2.11 (ICR 2.40, 95\% CI -0.17 to $4.97, P=0.07)$ for those who had used cannabis in the preceding year only. There was no evidence that abuse and cannabis use prior to (but not during) the preceding year combined synergistically to increase risk ( $\mathrm{ICR}=0.69,95 \% \mathrm{CI}$ -0.87 to $2.25, P=0.38$ ). All the main and synergistic effects reported here were evident for both paranoia and hallucinations, with no evidence of specificity (data not shown; available from the authors on request). 


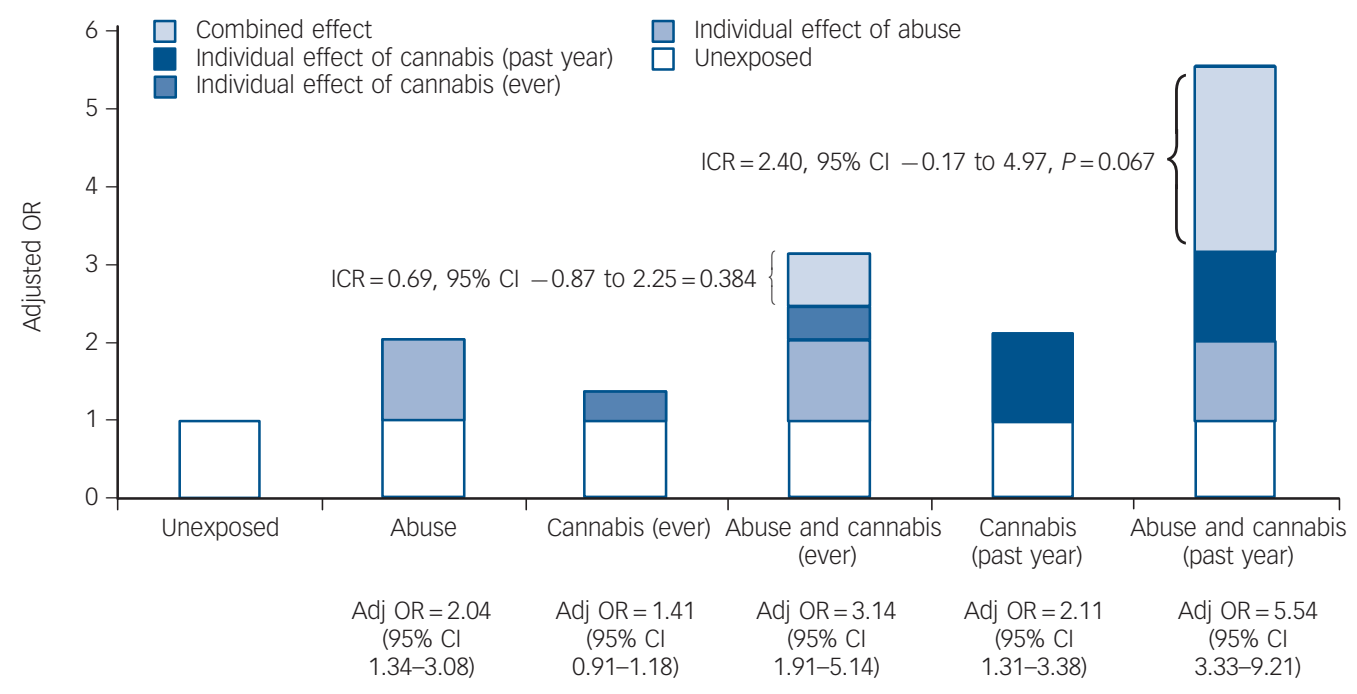

Fig. 3. Synergistic effects of abuse and cannabis use (ever and past year).

Odds ratio (OR) adjusted for age, gender, ethnicity, education and social class. Adj, adjusted; ICR, interaction contrast ratio.

\section{Discussion}

In these analyses, we sought to replicate and extend previous research by examining main effects of, and synergies between, childhood adversity, life events and cannabis use on odds of low-level psychotic experiences. A number of notable findings emerged. First, in relation to main effects, there was strong evidence that exposure to abuse and other adversities (life events) over the life course and recent cannabis use were associated with a two- to fourfold increased odds of reporting psychotic experiences in the 12 months prior to assessment. In addition, we found strong evidence that the impact of life events was cumulative and strongest for recent events that involved an element of threat or intrusion. Second, there was strong evidence that childhood abuse was associated with an increased odds of later life events and cannabis use, and, consistent with our hypotheses, there was evidence for synergistic effects of abuse and both life events and (more tentatively, given $P=0.07$ ) recent cannabis use on odds of psychotic experiences, such that the odds were greatest in those exposed to abuse and either life events or cannabis in the past year. Before considering these findings further, a number of methodological limitations need to be considered.

\section{Methodological limitations}

A number of potential limitations arise from the study design. Large-scale surveys inevitably have high levels of non-participation and necessarily involve collecting information retrospectively using instruments that can be administered relatively quickly by trained lay interviewers. Participation in SELCoH was in line with that in similar national surveys (for example the UK Adult Psychiatric Morbidity Survey ${ }^{31}$ ) and the sample was broadly representative of the local population on core demographic characteristics. ${ }^{18}$ We cannot rule out the possibility of selection bias arising from those with psychotic experiences being less likely to participate. $^{32}$ However, the high prevalence of psychotic experiences that we found in, and the broad representativeness of, our sample suggests this is unlikely to have been a major issue. Furthermore, Knudsen et $a l^{32}$ have shown that non-participation, although affecting prevalence and other population descriptors, has a limited impact on analytic, hypothesis testing. With regard to potential information bias, it is possible that presence of psychotic experiences lead to differential recall of events. ${ }^{33}$ However, none of those with psychotic experiences included in the analyses had received a diagnosis of a serious mental disorder, which suggests a higher rate of recall of events arising from an effort to explain the occurrence of disorder (i.e. effort after meaning) is unlikely to have influenced our findings. In addition, none of the interviewers were aware of any study hypotheses concerning psychotic experiences.

More significantly, the cross-sectional nature of our data inevitably limits the inferences that can be drawn regarding causation. We cannot, for example, rule out the possibility that some life events post-dated the onset of psychotic experiences and that some (for example separation from a partner) may have been a consequence of even low-level psychotic experiences, such as paranoia. This, however, is less likely to be the case for more distal life events and for childhood exposures. In the end, studies that can more robustly delineate the temporal sequence of exposure and outcome are required. Cross-sectional data such as that presented here, nonetheless, provide important pointers to the factors and mechanisms that may underlie the development of psychopathology.

There are further caveats to be noted concerning our assessments of psychotic experiences and of our primary exposures. For example, there are a number of limitations to the PSQ (and indeed other screening instruments) as a measure of psychotic experiences, and these have been well discussed in the past. ${ }^{34}$ It is likely, for example, that the PSQ, in not taking account of the context within which respondents endorse specific items, will overestimate the prevalence of psychotic experiences (e.g. some individuals may indeed be subject to deliberate efforts by others to harm them). Nevertheless, the consistency with which psychotic experiences, elicited using the PSQ, are associated with the same variables as for clinically significant psychosis strengthens the proposition that the PSQ does validly elicit unusual low-level psychotic experiences that represent perhaps the softest expression of an extended psychosis phenotype. In a similar vein, the information collected on our primary exposures was limited and did not include details on, for example, timing, duration and severity of adversities and events, nor were we able to determine the frequency, amount and type of cannabis use. This increases the likelihood of error. However, the effect of such misclassification would generally be to minimise differences between groups, such that reported effects are then likely underestimates. 


\section{Adversity, cannabis use and psychotic experiences}

The above limitations notwithstanding, the data and analyses reported here add further to the literature on psychotic experiences and the impact of environmental exposures. Most notably, our findings provide initial evidence that childhood adversity (i.e. abuse) and both life events and recent cannabis use may combine synergistically to increase the likelihood of psychotic experiences. Only a small number of previous studies have examined how environmental factors combine over time in relation to psychosis (for example Morgan et $a l^{13}$ and Clarke et $a l^{35}$ ). Bebbington et $a l^{36}$ for example, using data from the 2007 UK Adult Psychiatric Morbidity Survey (sample $n=7298$; with psychosis $n=38$ ), found some evidence that sexual abuse in childhood interacted with revictimisation (i.e. sexual abuse) in adulthood to increase risk for psychosis (defined as probable psychotic disorder). Lataster et $\mathrm{al}^{11}$ using data on composite measures of adversity from the Early Developmental Stages of Psychosis study (sample $n=1722$ ) of adolescents and young adults aged 14-24 years, found some evidence that early (i.e. baseline) adversity and a high level of recent (i.e. between baseline and around 4- to 8-year follow-up) adversity interacted on an additive scale to increase risk of low-level psychotic experiences at follow-up. Similarly, tentative evidence of synergistic effects of childhood adversity (variously measured) and cannabis use on broad psychosis phenotypes has been reported; ${ }^{12,37-39}$ the evidence on this, however, is not entirely consistent. ${ }^{40}$ Our findings are in line with, and add to, these emerging data in suggesting that the impact of both life events, particularly recent events, and cannabis use on likelihood of psychotic experiences depends, to a degree, on prior exposure to physical and sexual abuse. This is consistent with the hypothesis that childhood adversity creates, in some, a vulnerability for psychotic experiences that is realised in the event of exposure to further risk factors. Further, the evidence that childhood abuse is associated with a higher likelihood of exposure to these subsequent risk factors suggests that, for many, early adversity and abuse set in train a trajectory of repeated exposure to adversity and other factors that, over time, compound risk and push some along pathways to psychotic experiences and (more rarely) disorder - what we have elsewhere termed a sociodevelopmental pathway to psychosis. ${ }^{41}$

Our findings specifically in relation to life events further add to this. They suggest that life events have a cumulative impact on psychotic experiences and (more speculatively) that proximal events lead to a spike in risk that diminishes (but not entirely) over time. A similar interpretation is suggested by the finding that only cannabis use in the past year is associated with an increased likelihood of psychotic experiences. Of course, there is a need for caution in drawing too firm conclusions given the caveats noted above concerning uncertainty about the direction of effects. The data are nonetheless consistent with the hypothesis that it is repeated exposure over time, particularly to threatening and intrusive events, that increases risk and that risk waxes and wanes over time as a function, in part, of exposure to recent stressors and other risk factors such as cannabis use. It is tempting to speculate, then, that it is when exposures accumulate and continue over time that experiences and symptoms of psychopathology proliferate and risk of disorder, particularly psychotic disorder, amplifies - especially if occurring against a background of increased vulnerability. It bears noting, moreover, that despite speculation that life events increase risk for onset of psychosis, the number of studies with relevant data is surprisingly small. ${ }^{9}$ Our findings on life events are, therefore, particularly noteworthy.

\section{Next steps}

In so far as adverse social experiences tend to cluster in individuals, families and neighbourhoods and persist over time, it is essential to move on from identifying specific social and environmental risk factors for psychosis to examine the impact of multiple exposures, how they interact and the mechanisms through which they exert their effects. ${ }^{42}$ In doing this, we are currently constrained by limitations to available data and the intrinsic difficulties of disentangling cause and effect when the very outcome of interest shapes the social worlds of participants. These constraints notwithstanding, our findings provide initial support for the hypothesis that adversity and abuse in childhood create a vulnerability to psychotic experiences that becomes manifest as a consequence of further repeated exposure to other factors over time. This has potential implications for prevention and intervention (for example in identifying high-risk groups and intervening to promote resilience) and this in turn further supports the need for more studies using a range of designs and samples across the psychosis spectrum that examine possible (sociodevelopmental) pathways to psychosis through exposure to repeated adversity and other risk factors over the life course.

Craig Morgan, PhD, Ulrich Reininghaus, PhD, NIHR Biomedical Research Centre and Section of Social Psychiatry, Health Service and Population Research Department, Institute of Psychiatry, King's College London, UK: Abraham Reichenberg PhD, Department of Psychiatry, Mount Sinai School of Medicine, New York, USA; Souci Frissa, PhD, Matthew Hotopf, MBBS, PhD, Stephani L. Hatch, PhD, Psychological Medicine, Department, Institute of Psychiatry, King's College London, UK

Correspondence: Craig Morgan, Section of Social Psychiatry, Centre for Epidemiology and Public Health, Institute of Psychiatry, King's College London, UK. Email: craig.morgan@kcl.ac.uk

First received 27 Jun 2013, final revision 28 Oct 2013, accepted 13 Dec 2013

\section{Funding}

This research was supported by the Biomedical Research Nucleus data management and informatics facility at South London and Maudsley NHS Foundation Trust, which is funded by the National Institute for Health Research (NIHR) Mental Health Biomedical Research Centre at South London and Maudsley NHS Foundation Trust and King's College London and a joint infrastructure grant from Guy's and St Thomas' Charity and the Maudsley Charity. S.L.H., M.H., C.M. and S.F. receive salary support from the NIHR Mental Health Biomedical Research Centre at South London and Maudsley NHS Foundation Trust and King's College London. C.M. is further supported by funding from the Wellcome Trust (Grant Number: WT087417) and European Union (European Community's Seventh Framework Program (grant agreement No. HEALTH-F2-2009-241909) (Project EU-GEI)).

\section{Acknowledgements}

The SELCOH study team: Matthew Hotopf, Stephani L. Hatch, Souci Frissa, Maria Verdecchia, Aysha Begum, Robert Stewart, Nicola T. Fear, Abraham Reichenberg, Craig Morgan, Bwalya Kankulu, Jennifer Carter, Billy Gazard, Robert Medcalf, Alistair Baile, Elizabeth Doherty, Deborah Bekele, Helena Bundy, Joshua Buckman, Grant Henderson and Benedict Weobong.

\section{References}

1 Varese F, Smeets F, Drukker M, Lieverse R, Lataster T, Viechtbauer W, et al. Childhood adversities increase the risk of psychosis: a meta-analysis of patient-control, prospective- and cross-sectional cohort studies. Schizophr Bull 2012; 38: 661-71.

2 Morgan C, Fisher H. Environment and schizophrenia: environmental factors in schizophrenia: childhood trauma - a critical review. Schizophr Bull 2007; 33: 3-10.

3 van Dam DS, van der Ven E, Velthorst E, Selten JP, Morgan C, de Haan L. Childhood bullying and the association with psychosis in non-clinical and clinical samples: a review and meta-analysis. Psychol Med 2012; 42 2463-74.

4 Fisher H, Morgan C, Dazzan P, Craig TK, Morgan K, Hutchinson G, et al. Gender differences in the association between childhood abuse and psychosis. Br J Psychiatry 2009; 194: 319-25.

5 van Winkel R, Stefanis NC, Myin-Germeys I. Psychosocial stress and psychosis. A review of the neurobiological mechanisms and the evidence for gene-stress interaction. Schizophr Bull 2008; 34: 1095-105. 
6 Borges S, Gayer-Anderson C, Mondelli V. A systematic review of the activity of the hypothalamic-pituitary-adrenal axis in first episode psychosis. Psychoneuroendocrinology 2013; 38: 603-11.

7 Bentall RP, Fernyhough C. Social predictors of psychotic experiences: specificity and psychological mechanisms. Schizophr Bull 2008; 34: 1012-20.

8 Fisher H, Schreier A, Zammit S, Maughan B, Munafo MR, Lewis G, et al. Pathways between childhood victimisation and psychosis-like symptoms in the ALSPAC birth cohort. Schizophr Bull 2013; 39: 1045-55.

9 Beards S, Gayer-Anderson C, Borges S, Dewey ME, Fisher HL, Morgan C. Life events and psychosis: a review and meta-analysis. Schizophr Bull 2013; 39 740-7.

10 Moore TH, Zammit S, Lingford-Hughes A, Barnes TR, Jones PB, Burke M, et al. Cannabis use and risk of psychotic or affective mental health outcomes: a systematic review. Lancet 2007; 370: 319-28.

11 Lataster J, Myin-Germeys I, Lieb R, Wittchen HU, van Os J. Adversity and psychosis: a 10-year prospective study investigating synergism between early and recent adversity in psychosis. Acta Psychiatr Scand 2012; 125 388-99.

12 Harley M, Kelleher I, Clarke M, Lynch F, Arseneault L, Connor D, et al. Cannabis use and childhood trauma interact additively to increase the risk of psychotic symptoms in adolescence. Psychol Med 2010; 40: 1627-34.

13 Morgan C, Reininghaus U, Fearon P, Hutchinson G, Morgan K, Dazzan P, et al. Modelling the interplay between childhood and adult adversity in pathways to psychosis: initial evidence from the AESOP study. Psychol Med 2014; 44: 407-19.

14 Fisher HL, Caspi A, Poulton R, Meier MH, Houts R, Harrington $\mathrm{H}$, et al. Specificity of childhood psychotic symptoms for predicting schizophrenia by 38 years of age: a birth cohort study. Psychol Med 2013; 43: 2077-86.

15 van Os J, Linscott RJ, Myin-Germeys I, Delespaul P, Krabbendam L. A systematic review and meta-analysis of the psychosis continuum: evidence for a psychosis proneness-persistence-impairment model of psychotic disorder. Psychol Med 2009; 39: 179-95.

16 Kelleher I, Lynch F, Harley M, Molloy C, Roddy S, Fitzpatrick C, et al. Psychotic symptoms in adolescence index risk for suicidal behavior: findings from 2 population-based case-control clinical interview studies. Arch Gen Psychiatry 2012; 69: 1277-83.

17 Hatch SL, Frissa S, Verdecchia M, Stewart R, Fear NT, Reichenberg A, et al. Identifying socio-demographic and socioeconomic determinants of health inequalities in a diverse London community: the South East London Community Health (SELCOH) study. BMC Public Health 2011; 11: 861.

18 Hatch SL, Woodhead C, Frissa S, Fear NT, Verdecchia M, Stewart R, et al. Importance of thinking locally for mental health: data from cross-sectional surveys representing South East London and England. PLOS One 2012; 7: e48012.

19 Bebbington P, Nayani V. The Psychosis Screening Questionnaire. Int J Soc Psychiatr Res 1995; 5: 11-9.

20 Naroo JY. Ethnicity and Mental Health: Findings from a National Community Survey. Policy Studies Institute, 1997.

21 Singleton N, Bumpstead R, O'Brien M, Lee A, Meltzer H. Psychiatric Morbidity Among Adults Living in Private Households 2000. TSO (The Stationery Office) 2001

22 Morgan C, Fisher H, Hutchinson G, Kirkbride J, Craig TK, Morgan K, et al. Ethnicity, social disadvantage and psychotic-like experiences in a healthy population based sample. Acta Psychiatr Scand 2009; 119: 226-35.

23 Frissa S, Hatch SL, Gazard B, SELCoH study team, Fear NT, Hotopf M. Trauma and current symptoms of PTSD in a South East London community. Soc Psychiatry Psychiatr Epidemiol 2013; 48: 1199-209.
24 Rose M. Official Social Classifications in the UK. University of Surrey, 1998

25 Lewis G, Pelosi AJ, Araya R, Dunn G. Measuring psychiatric disorder in the community: a standardized assessment for use by lay interviewers. Psychol Med 1992; 22: 465-86.

26 Rothman KJ, Greenland S, Lash TL. Modern Epidemiology (3rd edn). Lippincott Williams \& Wilkins, 2008

27 Schwartz S, Susser E. Relationships among causes. In Psychiatric Epidemiology: Searching for the Causes of Mental Disorders (eds E Susser, S Schwartz, A Morabia, EJ Bromet): 62-74. Oxford University Press, 2006.

$28 \mathrm{Knol} \mathrm{MJ,} \mathrm{van} \mathrm{der} \mathrm{Tweel} \mathrm{I,} \mathrm{Grobbee} \mathrm{DE,} \mathrm{Numans} \mathrm{ME,} \mathrm{Geerlings} \mathrm{MI.} \mathrm{Estimating}$ interaction on an additive scale between continuous determinants in a logistic regression model. Int J Epidemiol 2007; 36: 1111-8.

29 Rothman KJ, Greenland S, Walker AM. Concepts of interaction. Am J Epidemiol 1980; 112: 467-70.

30 Kendler KS, Gardner CO. Interpretation of interactions: guide for the perplexed. Br J Psychiatry 2010; 197: 170-1.

31 Weich S, Brugha T, King M, McManus S, Bebbington $P$, Jenkins R, et al. Mental well-being and mental illness: findings from the Adult Psychiatric Morbidity Survey for England 2007. Br J Psychiatry 2011; 199: 23-8.

32 Knudsen AK, Hotopf M, Skogen JC, Overland S, Mykletun A. The health status of nonparticipants in a population-based health study: the Hordaland Health Study. Am J Epidemiol 2010; 172: 1306-14.

33 Susser $E$, Widom CS. Still searching for lost truths about the bitter sorrows of childhood. Schizophr Bull 2012; 38: 672-5.

34 Das-Munshi J, Bécares L, Boydell JE, Dewey ME, Morgan C, Stansfeld SA, et al. Ethnic density as a buffer for psychotic experiences: findings from a national survey (EMPIRIC). Br J Psychiatry 2012; 201: 282-90.

35 Clarke MC, Tanskanen A, Huttunen M, Leon DA, Murray RM, Jones PB. Increased risk of schizophrenia from additive interaction between infant motor developmental delay and obstetric complications: evidence from a population-based longitudinal study. Am J Psychiatry 2011; 168: 1295-302.

36 Bebbington $\mathrm{P}$, Jonas $\mathrm{S}$, Kuipers $\mathrm{E}$, King $\mathrm{M}$, Cooper $\mathrm{C}$, Brugha $\mathrm{T}$, et al. Childhood sexual abuse and psychosis: data from a cross-sectional national psychiatric survey in England. Br J Psychiatry 2011; 199: 29-37.

37 Houston JE, Murphy J, Adamson G, Stringer M, Shevlin M. Childhood sexual abuse, early cannabis use, and psychosis: testing an interaction model based on the National Comorbidity Survey. Schizophr Bull 2008; 34: 580-5.

38 Murphy J, Houston JE, Shevlin M, Adamson G. Childhood sexual trauma, cannabis use and psych osis: statistically controlling for pre-trauma psychosis and psychopathology. Soc Psychiatry Psychiatr Epidemiol 2013; 48: 853-61.

39 Konings $\mathrm{M}$, Stefanis $\mathrm{N}$, Kuepper $\mathrm{R}$, de Graaf R, ten Have M, van Os J, et al. Replication in two independent population-based samples that childhood maltreatment and cannabis use synergistically impact on psychosis risk. Psychol Med 2012; 42: 149-59.

40 Kuepper R, Henquet C, Lieb R, Wittchen HU, van Os J. Non-replication of interaction between cannabis use and trauma in predicting psychosis. Schizophr Res 2011; 131: 262-3.

41 Morgan C, Charalambides M, Hutchinson G, Murray RM. Migration, ethnicity, and psychosis: toward a sociodevelopmental model. Schizophr Bull 2010; 36: 655-64.

42 Hatch SL. Conceptualizing and identifying cumulative adversity and protective resources: implications for understanding health inequalities. J Gerontol B Psychol Sci Soc Sci 2005; 60 (special issue 2): s130-4.

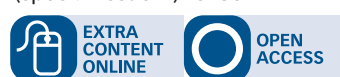

